logos_i_ethos_1_(32)_2012,s. 19-42

Krzysztof Skorulski

\title{
Ferdinand Ebner und der Platz der Dialogphilosophie in dem katholischen Denken des zwanzigsten Jahrhunderts ${ }^{1}$
}

Die Dialogphilosophie scheint in einigen Ländern nicht allzu oft in Zusammenhang mit dem katholischen Denken gebracht zu werden. Kann es also sein, dass in der katholischen Kirche nicht dialogisch gedacht wird, wie es manche moderne Theologen zu vermitteln scheinen? Wir wollen hier zeigen, dass genau das Gegenteil der Fall ist. Die Dialogphilosophie hat nicht nur in Ferdinand Ebner einen

Krzysztof Skorulski (1962) - geboren in Polen, lebt in Innsbruck. Promoviert in Theologie an der Universität Innsbruck, Aktivitäten in der Internationalen Ferdinand Ebner Gesellschaft, wo er gegenwärtig der Vorstand der Forschungssektion ist. Zahlreiche Übersetzungen ins Polnische u.a. der Werke von Henri Bergson und Wort und die geistigen Realitäten von Ferdinand Ebner. Einige Veröffentlichungen, vor allem in Polen im Bereich Philosophie und Spiritualität (Schwerpunkt Ferdinand Ebner).

katholischen Begründer gehabt, sondern sie hat auch im Herzen der Philosophie und Theologie des 20. Jahrhunderts ihren Platz gefunden.

Dieser Stand der Dinge ist zu unterstreichen, auch wenn ein Versuch, eine "katholische Dialogphilosophie" von dem protestantischen und jüdischen Denken abzusondern, mit Sicherheit scheitern würde. Das dialogische Denken ist nämlich vom Anfang an ein überkonfessionelles Phänomen. Wenn wir bei Ebner bleiben, müssen wir anmerken, dass, obwohl er seine Gedanken als erster formuliert, er dann auch Werke anderer "geistig verwandter” Denker liest: den Stern der Erlösung von Franz Rosenzweig, Ich und $D u$ von Martin Buber, Wesen und Formen 
von Sympathie von Max Scheler, Ich glaube an den dreieinigen Gott von Friedrich Gogarten; und er führt einen Briefwechsel mit Hans Ehrenberg. Einen integralen Teil dieser Überkonfessionalität bilden aber auch gleich vom „Ebner'schen” Anfang die katholischen oder, breiter gesehen, die christlichen Impulse: vor allem das „erst durch das Christentum bewusst gewordene" Ich und das von den Johanneischen Schriften inspirierte Konzept des Wortes.

\section{Dialogphilosophie und das dialogische Prinzip}

Am Anfang müssen wir zugeben, dass die Bezeichnung „Dialogphilosophie" nicht gerade durch Klarheit gekennzeichnet ist. Geht es dabei um eine Gruppe der Philosophen der ersten Hälfte des zwanzigsten Jahrhunderts, welche der „Dialogphilosophie” zugeordnet werden, manchmal ohne Rücksicht auf das, was sie tatsächlich schreiben? In diesem Kontext, neben den Namen von Buber, Rosenzweig, Levinas, Ebner (obwohl auch bezüglich ihrer Zugehörigkeit gelegentlich Zweifel gemeldet werden) werden unterschiedliche Denker genannt ${ }^{2}$. Oder geht es um ein philosophisches Nachdenken über das Phänomen des zwischenmenschlichen Dialogs (oder breiter: „der Begegnung”, bzw. „der Kommunikation”), das im Rahmen der Naturwissenschaften das Objekt der Kommunikationstheorie ${ }^{3}$, Kybernetik, Psychologie, Linguistik oder Literaturwissenschaft bildet? „Dialog” im Sinne der Kommunikation kann man aber auf verschiedene Weisen betrachten, nicht nur so, wie es unsere Denker tun, und wer weiß, ob eine gewisse Abwertung des Terminus „Dialog”, die wir aktuell beobachten, nicht mit dieser Unklarheit zusammenhängt. Um also die bisherigen Versuche, die Dialogphilosophie zu definieren ${ }^{4}$, zusammenzufassen, schlage ich

2 Urs von Balthasar erwähnt sogar T. Adorno i W. Benjamin als Dialogiker. Vgl. Ders., Theologik, Bd. 2: Wahrheit Gottes, Einsiedeln 1985, S. 42.

Vgl. P. Watzlawick und andere, Menschliche Kommunikation, Bern 1985.

Vgl. T. Gadacz, Historia filozofii XX wieku. Nurty, Bd. 2, Kraków 2009. Vgl. K. Wuchterl, Bausteine zu einer Geschichte der Philosophie des 20. Jahrhunderts. Von Husserl zu Heidegger. Eine Auswahl, Bern 1995, S. 364 f. 
vor, folgendes Kriterium aufzustellen: wenn wir annehmen, dass die Philosophie, oder genauer, gewisse philosophischen Einstellungen auch für das Betreiben jeglicher Wissenschaft notwendig sind, dann ist die Dialogphilosophie ein Denken, das als Grundlage das „dialogische Prinzip” hat, so wie es z. B. Ebner selbst am Anfang seines Hauptwerkes Das Wort und die geistigen Realitäten formuliert und als „Grundgedanken" benennt:

Vorausgesetzt, daß die menschliche Existenz in ihrem Kern überhaupt eine geistige, $[\ldots]$ so ist dieses wesentlich dadurch bestimmt, daß es vom Grund aus angelegt ist auf ein Verhältnis zu etwas Geistigem außer ihm, durch das es und in dem es existiert. Ein Ausdruck, und zwar eben der „objektiv” faßbare und darum einer objektiven Erkenntnis zugängliche Ausdruck des Angelegtseins auf eine derartige Beziehung ist in der Tatsache zu finden, daß der Mensch ein sprechendes Wesen ist, daß er das „Wort hat”. [...] Wenn wir nun, um ein Wort dafür zu haben, dieses Geistige im Menschen Ich nennen, das außer ihm aber, zu dem im Verhältnis das „Ich” existiert, Du, so haben wir zu bedenken, daß dieses Ich und dieses Du uns eben dur ch das Wort und in ihm in seiner „Innerlichkeit” gegeben sind; nicht jedoch als „leere” Wörter, denen kein Bezughaben auf eine Realität innewohnte - als was sie freilich in ihrem abstrakten, substantivierten und substantialisierten Gebrauche bereits erscheinen - vielmehr als Wort, das in der Konkretheit und Aktualität seines Ausgesprochenwerdens in der durch das Sprechen geschaffenen Situation seinen „Inhalt” und Realitätsgehalt „redupliziert”. Das ist in Kürze der Grundgedanke.

Den Sinn der Aussage Ebners kann man folgendermaßen formulieren: erstens gibt es kein reales „Ich” ohne „Du” - alle Arten des einsamen „absoluten Ich”, wie wir sie z. B. vom deutschen Idealismus kennen, sind lediglich intellektuelle Konstrukte, „leere Wörter”. Des Weiteren, diese „Ich und Du” - als Realitäten, nicht Fiktionen - sind uns gegeben, 2009, S. 14.

F. Ebner, Das Wort und die geistigen Realitäten. Pneumatologische Fragmente, Wien 
und zwar „objektiv gegeben” nicht anders als durch Wort und im Wort, aber im „Wort in der Aktualität seines Gesprochenwerdens” - d. h. im Gespräch, in der aktuellen Situation der Sprache. Ebner selbst sieht dieses Prinzip noch viel weiter, er schreibt: „In der Entdeckung des Ich und Du war aber nicht ein bloßes Prinzip des Denkens aufgedeckt worden, sondern eines des Lebens, das Prinzip des menschlichen Lebens in seiner Gottgewolltheit"6.

Er geht davon aus, dass der „Durchbruch”, der sich dabei in der menschlichen Einstellung vollzieht, nicht ohne Einfluss auf seine konkrete Existenz bleiben kann. Eine solche Philosophie bedeutete eine Revolution im Vergleich zum modernen „idealistischen” Denken, sie wäre ein „neues Denken” im Sinne Rosenzweigs ${ }^{7}$. Der Gegenstand der Reflexion wird dabei unerheblich, es geht immer mehr um ihr „Wie”. Auf diese Weise muss ein bestimmter Autor nicht „ausschließlich” Dialogphilosoph sein (d. h. einer bestimmten Gruppe angehören), er ist aber „auch” Dialogphilosoph, wenn er in seinem Denken das dialogische Prinzip verwendet, auf welchem Gebiet auch immer. Deswegen reden wir hier lieber von einem „dialogischen Denken” als von einer „Dialogphilosophie” im strengen Sinn.

Wenn wir mit dieser Definition der Dialogphilosophie einverstanden sind, dann müssen wir auch zugeben, dass schon die Darstellung der wichtigsten „dialogischen” und zugleich „katholischen” Denker unsere Skizze sprengen würde. Wir richten also unseres Interesse vor allem auf den „Entdecker” des dialogischen Prinzips, oder zumindest den, der daraus Konsequenzen gezogen hat - Ferdinand Ebner ${ }^{8}$, um danach auf seine Wirksamkeit bei zumindest ein paar innerhalb der katholischen Kirche bedeutsamen Gestalten aufmerksam zu machen.

$6 \quad$ Ders., Zum Problem der Sprache und des Wortes, [in:] Ders., Schriften, Bd. 1, München 1963, S. 646.

Vgl. F. Rosenzweig, Der Stern der Erlösung, Freiburg im Br. 1921, S. 660 f.

Ebner war zweifellos derjenige, der die letzten Konsequenzen dieses Prinzips gezogen hat, ihre Formulierung „hing in der Luft” bereits früher. Gadacz schreibt sie Jacobi zu. Vgl. T. Gadacz, Historia ..., op. cit., S. 503. Ähnliche Formulierungen - wie wir noch sehen werden - finden wir aber bereits bei Hamann oder Humboldt. 
Wir betonen jedoch, dass es uns hier nicht um das Aufweisen des „Einflusses” Ebners auf andere Denker geht (wir belassen es bei der Feststellung seiner zeitlichen Präzedenz). Unser Ziel ist lediglich das Hinweisen auf die Anwesenheit des dialogischen Prinzips im Denken des jeweiligen Autors. Dieses Prinzip kann als sein Leitgedanke oder Bezugspunkt funktionieren, auch wenn die von ihm erforschten Gebiete sehr breit sind und nicht nur Philosophie, sondern auch Theologie, Politikwissenschaft, Ökonomie oder gar Biologie oder Literaturwissenschaft umfassen ${ }^{9}$. Das dialogische Prinzip kann als eine Entdeckung solcher Art gesehen werden, dass sie danach als eine Grunddynamik des eigenen Denkens fungiert, so wie sich niemand offen auf Aristoteles beim Anwenden der Logik beruft.

\section{Das dialogische Denken vor Ebner}

Wie allgemein bekannt ist, erhebt sich die "dialogische Welle” im Denken der ersten Jahrzehnte des 20. Jahrhunderts, gewissermaßen als Antwort auf das „undialogische” Ereignis des Ersten Weltkriegs. Ebner kann also als Mitwirker an dieser Umwandlung des Denkens gesehen werden. Diese besteht erstens in einem Verwerfen des bisherigen idealistischen, auf der Stabilität der Strukturen basierenden Denkens. Stabiler als fallende politische, soziale oder kulturelle Strukturen erscheint der Mensch selbst. Es ist also kein Zufall, dass eine gewisse Denkweise, parallel zu den Gedanken Ebners, das Interesse solcher Denker wie Max Scheler und vor allem der Gruppe Rosenstock-Huessy, Cohen, Rosenzweig, Ehrenberg erweckt. Bald danach erweist sich, dass ähnliche Interessen auch Gogarten, Buber, Marcel und Guardini bewegen.

Dennoch wollen wir zuerst erörtern, ob bereits vor Ebner dialogische Ansätze im philosophischen Denken präsent sind. Jemand könnte nämlich fragen: Wenn das dialogische Prinzip tatsächlich die

9 Vgl. Die Themen der Vorträge in der Internationalen Ferdinand Ebner Gesellschaft, [in:] http://www.ebner-gesellschaft.org (3.02.2012). 
Grundlage des Menschenverständnisses bildet, warum blieb es bis $d a-$ to unbemerkt?

Nehmen wir gleich vorweg, dass, wenn wir mit unserem heutigen Wissen dialogische Spuren bei alten Philosophen finden wollen, wir nicht lange werden suchen müssen. Erwähnen wir nur die Klassiker. Der erste, der mit der Dialogizität in Verbindung gebracht werden muss, ist Platon mit seinen Dialogen (laut Ebner - Pseudodialogen, weil im Dienst der Maieutik). Spuren der uns interessierenden Denkweise stellen wir auch bei Augustinus fest: nicht nur in dem dialogischen Stil seiner Bekenntnisse ${ }^{10}$, sondern auch in dem Denken als unaufhörlichem Dialog mit dem „inneren Meister”: „Jener aber, der da befragt wird, lehrt, und das ist der, von dem es heißt, dass er im inneren Menschen wohnt, ist Christus"11. Der Zugang zur Wahrheit (heute könnte man sagen, zur Realität der anderen Person, hier konkret der Person Jesu) hängt von dem Vertrauen, Glauben und dem guten Willen ab, er „enthüllt sich jeweils nur so weit, wie der Mensch imstande ist, sie mit seinem geigenen Willen zu erfassen, gleichviel ob es sein guter oder böser Wille ist"12. Eine interessante Tätigkeit wäre es, nach den dialogischen Spuren beim Thomas von Aquin zu suchen. Ein interessanter Ansatz läge - meines Erachtens - in seiner Theorie der Engel: wenn Gott, laut Thomas, „über dem Universum” ist, dann muss der Mensch, um mit ihm zu kommunizieren, mit dem jeweils zu ihm gesandten Engel den Dialog führen ${ }^{13}$ - auch wenn es hier um einen speziellen inneren Dialog geht, sowie in seiner Theorie der Sakramente.

10 H. Urs von Balthasar sieht in Augustinus einen Denker „der ohne Konzession an der Grundeinsicht Ebners festhaltend sein zentrales Werk, die Confessiones, als eine Aussage seiner selbst zu Gott hin in Gebetsform verfaßt" (H. U. von Balthasar, Theologik, op. cit., S. 55).

11 Aurelius Augustinus, De magistro, 38, [in:] Der Lehrer. De magistro, übers. von C. J. Perl, Paderborn 1974, S. 84 f.

12 Ebenda.

13 Vgl. Thomas von Aquin, Summa theologica, I, 61, 3 ad 2: „Gott ist nicht ein Teil des Alls, sondern er ist über dem ganzen All; er besitzt, zuvor und auf überragende Weise, die ganze Vollkommenheit des Alls in sich. Der Engel aber ist ein Teil des Alls" (Ders., Summa theologica, T. 1, Paris 1872, S. 111). Vgl. Ders., Das Auge des Adlers, ein Brevier der Heilslehre, Zusammengestellt und verdeutscht von J. Pieper, München 1950, S. 129. 
Genauere Formulierungen bezüglich Dialogizität des Menschen finden wir bei Autoren wie Humboldt, Jean Paul, Schelling, Franz von Baader, von denen Ebner schreibt, dass in ihrer geistigen Umgebung bereits die „Entdeckung des Ich und Du” schwebte ${ }^{14}$. Humboldt schreibt zum Beispiel über das Wesen der Sprache:

Es liegt aber in dem ursprünglichen Wesen der Sprache ein unabänderlicher Dualismus, und die Möglichkeit des Sprechens selbst wird durch Anrede und Erwiderung bedingt. [...] Der Mensch sehnt sich, abgesehen von allen körperlichen und Empfindungs-Beziehungen, auch zum Behuf seines blossen Denkens nach einem dem Ich entsprechenden $\mathrm{Du}^{15}$.

Bei Baader lesen wir in einem Brief Folgendes über die Beziehung des Ichs zum Du:

Man hat, ja man gewinnt im Gemüt und Geist nur, was man gibt, und der Geber ist darum soviel Dank dem Empfänger schuldig als dieser jenem, d. $h$. Beide danken Gott, der im Geber wie im Empfänger derselbe ist. In dieser seligen Erkenntnis, dass es Einer und Derselbe ist, der in uns gibt und nimmt, der in uns sucht und findet, der in uns, durch und mit uns erhört und bittet, der das Licht und das Auge ist, in dieser seligen Erkenntnis, sage ich, löset sich das abstrakte, arme Ich und Du oder Nichtich in das reiche und sich genügende Wir auf... ${ }^{16}$.

Auch Ludwig Feuerbach, im Allgemeinen bekannt eher von seiner Kritik des Christentums, war bereits sehr nahe dran, das dialogische Prinzip zu formulieren. Ebner, der von dem Konzept Feuerbachs in den 1920er Jahren über Hans Ehrenberg erfuhr, schreibt:

14 Ebner schreibt in einem Brief an Josef Räuscher 16.01.1926: „Übrigens lag die «Entdeckung des Ich und Du» schon in der geistigen Luft um Humboldt, Jean Paul, Schelling, Baader herum" (F. Ebner, Schriften, Bd. 3, op. cit., S. 585).

15 W. von Humboldt, Ueber den Dualis, [in:] Schriften zur Sprachphilosophie, Bd. 3, Berlin 1843, S. 137.

${ }_{16} \quad$ Franz Baader und sein Kreis. Ein Briefwechsel, Hrsg. F. Werle, Leipzig 1924, S. 185. 
Feuerbach also entdeckte die Beziehung des Ichs zum Du, die wesentliche Gebundenheit des Selbstbewußtseins an das Bewußtsein eines Du; den „objektiven” Träger dieser Beziehung aber im Wort zu sehen, war ihm noch verwehrt ${ }^{17}$.

In diesem Kontext sollen wir noch Kierkegaard erwähnen, dem Ebner eine besondere Gabe zuschreibt, den Leser seiner Schriften zum „konkreten” Du zu machen ${ }^{18}$. Interessant ist, dass weder Hamann, noch Humboldt oder Feuerbach unter ihren Zeitgenossen ein besonderes Echo erwecken konnten. Der Theologe Hans Urs von Balthasar schreibt bezüglich Hamann: „es brauchte anderthalb Jahrhunderte, bis Ebner, Buber, Haecker seine Sprachtheologie wieder entdeckten"19.

Es kann sein, dass die Philosophie vor dem 20. Jahrhundert den Dialog nicht zum Thema machte, da sie immer noch die großen Probleme aus der Perspektive des einsamen denkenden Subjektes (das Kartesianische „Ich denke, also bin ich”) lösen wollte. In der Einstellung der einzelnen Denker merken wir einen großen Unterschied zwischen dem, was die „ernsthafte” Philosophie beschäftigen darf, und dem, was man - wie der bereits zitierte Baader - in einem Brief an eine junge Frau schreiben kann. Ein Dialog konkreter Personen ist durch eine Dialektik der Ideen ersetzt worden. Zum Übersehen der Problematik des Ich und Du hat auch zweifellos die Tatsache beigetragen, dass es, um dialogisch zu denken, ein richtiges Konzept des Ich (oder der Person) braucht, ein Konzept eines abstrakten Subjektes reicht hier nicht aus. Kein Wunder, dass Ebner eine solche Philosophie kritisiert und ihr Ende verkündet ${ }^{20}$.

\section{Ferdinand Ebner und sein Konzept des Wortes}

Im Ebner'schen Denken hat das Konzept des Wortes einen zentralen Wert. Darin sieht Ebner den entscheidenden Schritt zur Formulierung

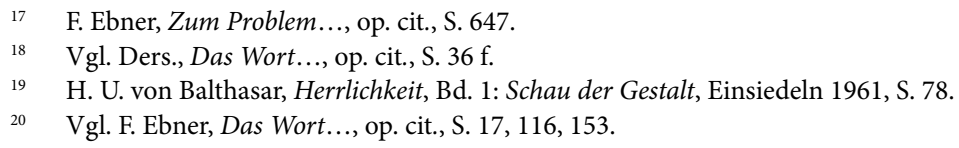


des dialogischen Prinzips selbst. Beim Präsentieren dieses Prinzips haben wir bereits angedeutet, dass Ich und Du objektiv im Wort und durch Wort gegeben sind. Das Konzept des Wortes ermöglicht es uns, den Menschen überhaupt zu verstehen, auch in seinem Verhältnis zu Gott. In unseren Analysen werden wir uns also vor allem auf das Ebner'sche Konzept des Wortes konzentrieren, das uns als der bedeutendste Beitrag des österreichischen Philosophen zum Verständnis der Dialogik erscheint.

\subsection{Das „richtige” und das „technische” Wort}

Ebner unterscheidet vor allem zwischen dem Wort im „technischen” Sinne (Wort mit dem Plural „Wörter”) und dem „rechten Wort” (Wort mit dem Plural „Worte"). Das erste ist eine durch die Linguistik zu analysierende Spracheinheit, ein Zeichen im Zeichensystem, welches die Sprache bildet. Diese Art von „Wörtern” kann man mit den Begriffen gleichsetzen. Ebner spricht von ihnen als von „toten Wörtern”. Aber nicht dieses Wort interessiert unseren Gablitzer Denker. Ihm geht es vor allem um das Wort in dem zweiten Sinn, um ein „lebendiges und sinnvolles” Wort: dieses Wort ist ein gesprochenes Wort, es ,ereignet sich” im Alltag, hat eine wirksame Kraft. Wenn ich an jemanden mein Wort richte, bedeutet das, dass ich mit ihm in Beziehung trete. Das Wort in diesem Sinne ist also ein Satz, die Sprache, und der Ursatz (Urwort) ist laut Ebner „Ich bin”. Nur dieses dialogische Wort hat eine "geistige Bedeutung"21, indem es „sowohl das Sein und den Sinn des Ich als auch des Du, also die «geistigen Realitäten», objektiv wahrnehmbar" macht ${ }^{22}$.

Wie ich woanders erläutert habe, galt die Leidenschaft Ebners der Überwindung des monologischen, mit der Idee verbundenen Denkens, welches von ihm „Idealismus” genannt wurde, indem er ihm ein dialogisches, mit dem Wort verbundenes Denken entgegensetzt. Der Idealismus, als ein monologisches Denken, setzt "Selbstgenügsamkeit eines Bewusstseins in allen Bereichen des Lebens". Dem Idealisten reicht ein (genau gesagt: das

${ }_{21} \quad$ Ders., Zum Problem..., op. cit., S. 646.

22 Vgl. ebenda. 
eigene) Bewusstsein, während das dialogische Denken immer mehr als ein Bewusstsein braucht ${ }^{23}$. Und genau hier ist der Platz für das „,richtige Wort”.

\subsection{Das Wort und die Person}

Das charakteristische Merkmal des Menschen ist laut Ebner, dass er „das Wort hat" ${ }^{24}$. Dies bedeutet, das er ein Du ansprechen und von dem Du angesprochen werden, also das Du für ein anderes Ich werden kann. Auf diese Weise ist die Aussage „der Mensch hat das Wort” gleichbedeutend damit, dass der Mensch „auf ein Verhältnis zu etwas Geistigem außer ihm” „vom Grund aus angelegt ist”25. „Das Wort haben” heißt auch „Person sein”. Beim Beschreiben des Unterschiedes zwischen der Existentialaussage in der ersten oder zweiten und der in der dritten Person, formuliert es Ebner folgend:

Diese gedanklich nicht aufzuhebende Identität des Subjekts der Aussage und des Prädikats macht das Wesen der Existentialbehauptung in der ersten und zweiten Person aus, in der eben ein Sein im Sinne der Personalität behauptet wird, das an und für sich die Beziehung zur Aussage, zum Wort in sich begreift, weil Persönlichkeit und „Wort haben” ein und dasselbe sind ${ }^{26}$.

Daraus kann man schließen, dass Person zu sein heißt: „Ich bin” oder „Du bist” sagen zu können - und zwar so, dass diese Aussage (in der Anwesenheit des $\mathrm{Du}$ ) sinnvoll ist und im Aufbau einer Beziehung resultiert.

\subsection{Das Wort in der Aktualität seines Gesprochenwerdens}

Die Aussage „Ich bin” zeigt nämlich ihren eigentlichen Sinn, wird zum „rechten Wort” erst dann, wenn das Wort in der Aktualität seines

23 Vgl. T. Fedyayeva, Dialog i satira, Sankt-Peterburg 2004, S. 6, 32.

24 F. Ebner, Das Wort..., op. cit., S. 14 f, 19 u. a.

25 Ebenda, S. 14.

26 Ebenda, S. 138. 
Gesprochenwerdens verstanden wird. Erst dann sieht man auch den Unterschied zwischen Wort und Begriff.

Die Aktualität eines zwischen Personen gesprochenen Wortes (z. B. „Ich bin”), wird dann gewährleistet, wenn beide anwesend und in Übereinstimmung bleiben, ihre Worte wahrhaft und ernst sind. Man kann dabei auch von einem gegenseitigen Vertrauen oder dem Glauben an das Wort reden. Durch den Glauben an das Wort werden beide Personen zu einer Art „Interaktion” mobilisiert, zum gemeinsamen Handeln. Die Funktion des Wortes ist es also, Personen in Beziehung zu bringen, das Verhältnis des Ich zum Du aufzubauen. Wenn ich in einer solchen Situation, „aktuell”, das Wort an eine andere Person richte, dann denkt diese nicht an die „sprachlichen Zeichen”, sondern sie glaubt an dieses Wort und handelt auch entsprechend. Ohne diese Aktualität kann man das Wort zerstückeln und (z. B. grammatikalisch) analysieren ${ }^{27}$ - das tut man aber nicht vor einem anderen Menschen.

\subsection{Das Wort und die Liebe}

Wir haben bereits angedeutet, dass mit der Aktualität des Wortes das Vertrauen zusammenhängt. Ebner formuliert es noch radikaler und spricht in diesem Kontext von der Liebe. Für ihn ist das Wort kein „rechtes Wort” ohne Liebe:

So gehören denn das Wort und die Liebe zusammen. Zwar schöpft der Mensch aus jenem und der durch es in ihn gelegten Vernunft alle Kraft des Erkennens, im letzten Grunde jedoch seines Ihm-Gegebenseins dient es der Liebe, der göttlichen Liebe und der von Gott geforderten Nächstenliebe. Das rechte Wort ist immer eines, das die Liebe spricht, und es wohnt ihm die Kraft inne, chinesische Mauern zu durchbrechen. [...] Das lieblose Wort aber ist bereits

27 Merken wir, dass z. B. die Analysen der Dekonstruktivisten beziehen sich genau auf dieses „Wort ohne Aktualität” als „texte”. Vgl. R. Brütting, „Écriture” und „texte”. Die französische Literaturtheorie "nach dem Strukturalismus”. Kritik traditioneller Positionen und Neuansätze, Bonn 1976 , S. 73 f. 
menschlicher Mißbrauch mit der göttlichen Gabe des Wortes. In ihm streitet das Wort wider seinen eigenen Sinn und hebt geistig sich selbst auf ${ }^{28}$.

Die Realitäten des geistigen Lebens, d. h. Ich und Du, haben also für Ebner im Wort ihre objektive und in der Liebe ihre subjektive Existenz. Er stellt es folgendermaßen dar: „Dessen Objektiv-im-Wort-Gegebensein entspricht sein «subjektiver» Bestand in der Liebe; so daß also das Wort und die Liebe in ihrem geistigen Grunde zusammengehören"29. Um die Mission des Ins-Verhältnis-Setzens des Ich zum Du zu erfüllen, reicht also nicht „irgendein” Wort, es geht um das aktuelle und mit Liebe verbundene Wort, um das „rechte Wort”.

Die Liebe wird dabei sehr weit verstanden, als Offenheit und Respekt gegenüber dem Anderen (so wie er ist), als eine positive Einstellung ihm gegenüber. Ohne Liebe verbindet mich das Wort nicht mit dem Anderen als Du, und es kann sogar töten. Bedenken wir also, dass Ebner - wie bereits viele Denker vor ihm ${ }^{30}$ - annimmt, dass die Offenheit des Menschen, also die genannte Liebe, eine Haltung ist, die sowohl seine Einstellung zu sich selbst, als auch zum Anderen und sogar $\mathrm{zu}$ Gott umfasst. Kurz gesagt, man kann nicht andere Menschen lieben ohne sich selbst zu lieben, ohne Gott zu lieben. Diese positive Einstellung dem Anderen gegenüber ist nur subjektiv feststellbar, gewissermaßen „von innen”, denn von außen haben wir zu ihr nur in den Worten und dem Verhalten der betreffenden Person Zugang.

\subsection{Das Wort im Anfang}

Wie ist es dazu gekommen, dass der Mensch überhaupt „das Wort hat"? Diese Frage verbindet sich für Ebner mit der Frage nach dem Anfang der Sprache. Seine Überlegungen führen ihn zu einem anderen Aspekt des

28 F. Ebner, Das Wort..., op. cit., S. $92 \mathrm{f}$.

29 Ebenda, S. 25.

30 Diese Ansicht finden wir z. B. bei Thomas von Aquin, vgl. Summa theologica I, 117, 2 wo er von DER Liebe spricht, die alle Bereiche durchwirkt. Vgl. Thomas von Aquin, Das Auge des Adlers..., op. cit., S. 132. 
Wortes, zum „Wort im Anfang”. Inspiriert durch den Prolog des Johannesevangeliums verbindet er das "rechte Wort" mit dem biblischen Logos.

Wer spricht als erster das Wort aus? Wer macht mich zur Person? Der Mensch merkt, dass er immer schon angesprochen ist; bevor er zum Ich wird, ist er zuerst das Du eines Ich. Dieses Ich, das den Menschen anspricht, ist also für Ebner letzten Endes Gott. Um in der Linie des biblischen Denkens zu bleiben: es ist Jesus Christus, der das an den Menschen gerichtete Wort Gottes ist. Wenn das Wort das "Vehikel” ist, in dem ich objektiv den Anderen wahrnehme, dann ist auch Jesus Christus als Wort eine Möglichkeit, Gott wahrzunehmen. Ein ähnliches Denken erfüllt die Ikonenmalerei: wir finden dort kaum Abbildungen Gottes des Vaters, sondern nur Jesus Christus, denn er hat ja selbst gesagt „Wer mich sieht, sieht auch den Vater" ${ }^{31}$. Ebner geht es aber - wie bereits gesagt - nicht nur ums Denken, sondern ums Leben. Er betont also: das im Wort - dem Wort Christi im Evangelium - ist dem Menschen die Wirklichkeit Christi gegeben, die beim Anwenden dieses Wortes im eigenen Leben erfahren werden kann ${ }^{32}$.

\section{Dialogphilosophie im katholischen Denken des 20. Jh}

Unter den wichtigsten katholischen Autoren, die in ihrem Denken das dialogische Prinzip verwendeten, nehmen wir hier nur solche ins Visier, die einen direkten Bezug auf Ebner zugeben. Aus Platzgründen können wir in unserer Skizze weder ihre Konzepte ausführlich darstellen, noch zeigen, in welchen Punkten sie dem dialogischen Prinzip treu bleiben und in welchen nicht. Es geht uns vielmehr darum zu zeigen, dass der dialogische Gedanke auch die zentralen Strömungen der philosophischen und theologischen Überlegungen des 20 Jahrhunderts auf eine diskrete Weise durchwirkt.

$31 \quad$ Hier können wir diesen wichtigen Gedanken Ebners nicht genauer besprechen. Eine ausführliche Darstellung dieses Themas finden wir z. B., [in:] J. Jagiełło, Vom ethischen Idealismus zum kritischen Sprachdenken, München 1997, S. 230-298.

32 Vgl. F. Ebner, Die Wirklichkeit Christi, „Der Brenner”, 1926, nr 10, S. 44 f. 


\subsection{Romano Guardini}

Die Bedeutung Romano Guardinis innerhalb der Erneuerungsbewegung in der katholischen Kirche des 20. Jahrhunderts braucht nicht eigens betont zu werden. Aufgrund der Vielfalt seiner Interessen, der Vielzahl seiner Aktivitäten und Schriften lässt er sich nicht „einfach” als Dialogphilosoph bezeichnen, dennoch berücksichtigt Guardini in seinem Denken über Mensch und Gott das dialogische Prinzip.

Guardini geht in seinen anthropologischen Überlegungen davon aus, dass die bisherige Sicherheit unseres Wissens über den Menschen, sowohl des humanistisch-geistenwissenschaftlichen als auch des naturwissenschaftlich-technischen, erschüttert worden ist, was eine ernste Frage nach dem Menschen ermöglicht. Er versucht ein „christliches Menschenbild" zu entwerfen, das über alle bisherigen Antworten auf diese Frage hinausgeht, und das sich in dem Konzept der „Person” ausdrückt. In diesem Bild tut man dem Menschen nicht genug, indem man auf seine Innerlichkeit hinweist, auch wenn diese als Selbstbewusstsein, als die Quelle des Willens, und des Handelns erfasst wird. Nach einer Person kann man auf zwei Weisen fragen. Die erste ist „Was ist das da?”. Die Antwort würde dann lauten: „Ein gestaltetes, in Innerlichkeit begründetes, geistig bestimmtes und schaffendes Wesen..." Wenn wir aber fragen: „Wer ist Dieser da?” - ist die Antwort „Ich”. Guardini schreibt: „Jetzt erst ist die Person berührt”33. Die Person kann also nur durch eine andere Person bedingt und nur von ihr abhängig sein, nicht durch irgendwelche äußeren Umstände. Diese Bedingung wird aber nicht durch die Objekt-Subjekt Beziehung bestimmt, „zum Du wird nur der Andere [vielmehr] erst dann, wenn die einfache SubjektObjekt Beziehung aufhört" ${ }^{34}$. Guardini sieht den Menschen also als Person, die ,in der Form des Dialogs, auf die andere Person hingeordnet besteht. Sie ist wesentlich dadurch bestimmt, Ich eines Du zu werden.

33 R. Guardini, Welt und Person, Versuche zur christlichen Lehre von Menschen, Würzburg 1940, S. 94.

${ }^{34}$ Ebenda, S. 106. 
Die grundsätzlich einsame Person gibt es nicht" ${ }^{35}$. Hier sehen wir also die Anwendung des dialogischen Prinzips. Auch bezüglich der Sprache finden wir zwischen Guardini und Ebner deutliche Parallelen: der Mensch hat die „Gabe der Rede”, und die Sprache setzt die Existenz des Anderen voraus, das Sprechen drängt ihn zur Verwirklichung der Beziehung Ich-Du. „Damit bildet die Sprache den objektiven Vorentwurf für das Zustandekommen der personalen Begegnung" ${ }^{36}$. Auch Gott hat den Menschen „durch Anruf erschaffen” ${ }^{37}$.

Diese offensichtlichen Ähnlichkeiten zwischen diesen Gedanken und den Formulierungen Ebners haben Guardini - der normalerweise seine Bezugspunkte nicht verrät - sogar dazu bewegt, in dieser Materie Stellung zu nehmen. Er schreibt: „Wie mir gesagt wurde, sind die dargelegten Gedanken denen verwandt, die Ferdinand Ebner entwickelt hat" ${ }^{38}$. Wir lassen hier die Frage nach dem tatsächlichen Einfluss Ebners auf Guardini beiseite, merken aber, dass Guardini als einer der ersten, noch in den 20er Jahren, das im Denken Ebners vorhandene Potential entdeckte, das der Erneuerung des Denkens in der katholischen Kirche helfen könnte. Daher ließ er Ebners' Werk nicht nur gelten, sondern er bot ihm auch eine Zusammenarbeit im Rahmen der von ihm damals redigierten Zeitschrift „Schildgenossen” an. Diese Arbeit, kam jedoch aufgrund der Krankheit Ebners schließlich nicht zustande.

Ausgehend von seinem dialogischen Verständnis der Person (als eine der Grundlagen seiner „christlichen Weltanschauung”) kann Guardini seine Konzepte auf so breiten Feldern entwickeln wie die politische

$\begin{array}{ll}35 & \text { Ebenda, S. } 112 . \\ 36 & \text { Ebenda, S. } 109 . \\ 37 & \text { Ebenda, S. 114. }\end{array}$

38 Ebenda, S. 114 f. Guardini betont hier seine Selbständigkeit: „Ich habe aus Ebners Schriften nur vereinzelte Stücke gelesen. So freue ich mich der Übereinstimmung, darf aber das Entwickelte ohne Berufung auf ihn stehen lassen. Wirkliche Anregungen verdanke ich Theodor Haecker, wüßte aber nicht anzugeben, durch welche Schrift oder Äußerung”. Sagen wir dazu, dass selbst Theodor Haecker ein Sympathisant Ebners' war, er hat ihn auch in den „Brennerkreis” eingeführt und de facto die Veröffentlichung der Fragmente ermöglicht. Auf der anderen Seite waren es die Schriften Haeckers über Kierkegaard, die Ebner in seinem Denken halfen. 
Ethik („das demokratische Prinzip” ${ }^{39}$ ), die Offenbarung (als die „Ansprache" des Menschen von Gott) oder die Liturgie (Christozentrismus - Jesus Christus als das Wort Gottes an den Menschen). Gerade diese Vorgangsweise, d. h. der Anfang bei der Analyse der einzelnen Phänomene und dann die Stellungsnahme dazu aus dem Gesichtspunkt der "christlichen Weltanschauung” wird als sein besonders starker Punkt angesehen ${ }^{40}$. So können wir an Guardini ein klassisches Beispiel, vielleicht nicht des „Dialogphilosophen”, aber auf alle Fälle des am Dialogprinzip orientierten Denkers darstellen.

\subsection{Gabriel Marcel und der Personalismus}

Der Einfluss einer Gestalt wie Gabriel Marcel in den katholischen Kreisen kann kaum überschätzt werden. Um ihn jedoch mit den deutschsprachigen Autoren zu vergleichen, müssen wir zuerst allgemein feststellen, dass das dialogische Denken in Frankreich einen eigenen, wenn auch zum Deutschen parallelen Weg beschritt. Die Richtungen, in deren Rahmen dialogisch gedacht wurde, heißen hier „der christliche Existentialismus” und „Personalismus" ${ }^{21}$. Kein Wunder also, dass Marcel, in der

39 Das demokratische Prinzip: „Das Dasein ruht auf einer Vielheit von Personen; auf der Mannigfaltigkeit ihrer Anschauungen, ihrer sozialen und kulturellen Impulse. Diese Vielheit ist berechtigt und darf ihr Recht durch selbstbestimmte Lebensführung und Arbeitsleistung beweisen; so kann eine Einheit immer nur durch ebenfalls freies Zusammenwirken zu Stande kommen. Also muß Jeder den Anderen anerkennen, ihm Raum geben, seine Überzeugung als solche achten, auch wenn er sie nicht teilt, und stets bereit sein, mit ihm, wo nur möglich, in Kooperation zu treten" (R. Guardini, Pluralität und Entscheidung, [in:] Ders., Sorge um den Menschen, Würzburg 1962, S. 137).

40 Vgl. W. Dettloff, Romano Guardini (1885-1968), [in:] Klassiker der Theologie, Bd. 2, München 1983, S. 323.

${ }^{41}$ Vielleicht wird gerade deswegen, zusätzlich nach dem Erscheinen der Schriften von Levinas die Bezeichnung „Dialogphilosophie” in einigen Ländern vor allem mit der Gruppe jüdischer Denker in Verbindung gebracht, so dass z. B. T. Gadacz für den polnischen Leser betonen muss, dass „die Dialogphilosophie nicht nur, wie die gängige Meinung behauptet, eine jüdische, sondern gleichermaßen eine christliche Philosophie war" (T. Gadacz, Historia..., op. cit., S. 503). Gleichzeitig wird Ebner (zusammen mit Buber) ein „dialogischer Personalist” genannt. Vgl. J. Ratzinger, Dogmatische Konstitution über die Göttliche Offenbarung Dei Verbum, [in:] Das Zweite Vati- 
französischen philosophischen Tradition aufwachsend, andere Termini für das dialogische Prinzip verwendet. Daher wird er nicht immer - abgesehen davon, dass er ein vielfältiger, schwer einzuordnender Denker ist - zu den Dialogikern gezählt. Marcel wird - übrigens ähnlich wie oft Ebner $^{42}$ - am ehesten als ein „christlicher Existentialistbezeichnet”, wobei er selbst sich am liebsten „christlicher Sokratiker” nennt.

Obwohl sich Marcel nur ungern „Existentialist” nennen lässt ${ }^{43}$, richtet er sich in seinem Denken nicht auf eine Idee, sondern - wie er selber sagt - „konkret auf die Existenz, und nicht nur auf meine Existenz, sondern auch auf das Du" ${ }^{\prime 4}$. Auch er verwirft das ,intentionale" Modell für die zwischenmenschliche Begegnung: „Der Andere als solcher, als existierend, ist für mich nicht Objekt; er kann natürlich zum Objekt werden. Dies ist immer eine Gefahr, ein Risiko”. „Das Existentielle” nennt Marcel „Intersubjektivität”, „wir zusammen”. Wenn wir aber feststellen, dass „die Intersubjektivität für Marcel das Wesen der Person ist”, oder, anders gesagt: „Der Mensch strebt Gemeinschaft an” ${ }^{45}$, so entdecken wir das dialogische Prinzip als Grundlage seines Denkens.

Eine radikale Einsamkeit, verstanden als Verschlossensein gegenüber anderen Menschen, ist für Marcel gleichbedeutend mit Selbstmord (metaphorisch, aber auch wörtlich verstanden). Die Unterscheidung zwischen „Ich” und „meinem Leben” - von der die Absicht, das Ich um sein Leben zu bringen, starten muss - geschieht nämlich in der

kanische Konzil: Dokumente und Kommentare. 3 Ergänzungsbände zum „Lexikon für Theologie und Kirche”, Hrsg. H. Vorgrimmler, T. 2, Freiburg 1967, S. 506.

${ }_{42}$ Man braucht nur die Ergebnisse der Google-Suche nach „Ferdinand Ebner” im Internet anzuschauen. Vgl. M. Kobayashi, Das Wort und die Liebe im christlichen Existentialismus Ferdinand Ebner, [in:] http://www.sed.tohoku.ac.jp/library/nenpo/contents/15/15-03.pdf (3.02.2012); F. Heer, Der Philosoph aus dem Cafe Lehn, [in:] http://www.noekulturforum.at/frames/texte/ show_1_text.php?p_id=2\&buchstabe=alle\&suchwhat=autor (3.02.2012); oder auch das deutsche Artikel in Wikipedia, vgl. http://de.wikipedia.org/wiki/Ferdinand_Ebner (3.02.2012).

${ }_{43}$ Die Auffassung, er „sei ein Exponent des christlichen Existentialismus” bezeichnet Marcel als „ein Missverständnis, für das Sartre an erster Stelle verantwortlich ist... Das ist sinnlos. Das Wort Existentialismus habe ich selbst nie gebraucht" (G. Marcel, Mein philosophisches Testament, [in:] Ders., Werkauswahl, Bd. 3: Unterwegssein, Paderborn 1992, S. 320).

${ }_{44}$ Ebenda, S. 324.

45 P. Grotzer, Einleitung, [in:] G. Marcel, Werkauswahl, op. cit., S. 9. 
Einsamkeit. „Wer abdankt, wer das Leben verabschiedet, sollte erkennen, dass seine Haltung ein praktisches Selbstgenügen einschließt" ${ }^{\prime \prime}$. Wenn wir sagen, dass das Leben eine Gabe ist (also eine Beziehung voraussetzt), dann sind wir auch dafür verantwortlich. Wenn ich mich als eine Monade betrachte, verschwindet diese Verantwortung.

Einen Zugang zum Wesen des Anderen wird mir nicht in der Intentionalität geboten, sondern in der „Disponibilität” (disponibilité), in einem bedingungslosem „sich auf etwas [auf Dich - K. S.] verlassen, was mir nie objektiv sein wird”. Auf diese Weise können wir von „Haben” zum „Sein” übergehen, welches wahrhaft menschliche Bezüge charakterisiert.

Auch Marcel berücksichtigt in seinen phänomenologischen Analysen verschiedene menschliche Gebiete, hält aber zugleich an dem dialogischen Prinzip fest. Zu den für ihn wichtigen und oft wiederkehrenden Themen gehören der Körper und Körperlichkeit ${ }^{47}$, Hoffnung, Glaube (,glauben im strengen Sinn [...] [heißt] immer glauben an ein $\mathrm{Du}$, an eine persönliche Realität, die angerufen werden kann"48), oder Treue.

Gabriel Marcel ist also ein mit Ebner ,geistesverwandter” Denker, der übrigens dessen Beitrag und zeitliche Präzedenz (neben Rosenzweig) auf dem Gebiet der Dialogphilosophie betont ${ }^{49}$. Vielleicht - wie Zucal es suggeriert - geht eine Ebner'sche Inspiration im Denken Marcel' sogar noch weiter, als er es selbst zugibt, z. B. wenn wir an sein Streben denken, die Ebene des „Habens” in der Liebe zu transzendieren, in der Liebe für die der Andere kein Objekt („Er”) mehr ist, sondern ein im Dialog erfahrbares $\mathrm{Du}^{50}$. Natürlich zeigt auch Marcel selbst - indem

46 G. Marcel, Mein Leib, mein Sein, mein Leben, [in:] Ders., Dialog und Erfahrung, Frankfurt a. M. 1969, S. 122.

47 Auch wenn er in seinem „philosophischen Testament” sagt: „,ich neige heute zu den Ansicht, dass es zweifellos fruchtbarer ist, über mein Leben nachzudenken, als über meinen Leib” (also ähnlich wie Ebner), schließlich, „spreche ich ja von einem Leib in seiner lebendigen Wirklichkeit, im Gegensatz zu dem leblosen Körper" (ebenda, S. 117).

48 G. Marcel, Schöpferische Treue, [in:] Ders., Werkauswahl, op. cit., S. 201.

49 Vgl. Ders. Die geistige Entwicklung Ferdinand Ebners, [in:] Ders., Dialog und Erfahrung, op. cit., S. $111 \mathrm{f}$.

50 Vgl. S. Zucal, Ferdinand Ebner, la „nostalgia” della parola, Brescia 1999, S. 196. 
er seine eigene Originalität betont - einige Unterschiede zu Ebner. Zum Beispiel distanziert er sich von dem „radikalen Christozentrismus Ebners", der - wie er suggeriert - in dem Übersehen des menschlichen Du besteht, um sich im Menschen direkt an Christus zu wenden ${ }^{51}$. Einen anderen Unterschied bildet die von Marcel entwickelte (und für Ebner sicher inakzeptable) Philosophie des „Wir, in der Ich und Du eine gewisse metaphysische Einheit zu bilden scheinen" ${ }^{2}$.

Wie wir bereits angedeutet haben, parallel zu der meistens deutschsprachigen Dialogphilosophie haben wir es mit der Entwicklung einer anderen, verwandten (auch wenn mit gewissen Unterschieden) vorwiegend französichssprachigen Richtung zu tun. Es geht um den Personalismus. Es ist nicht unsere Aufgabe, Ähnlichkeiten und Unterschiede beider Richtungen zu analysieren; dennoch merken wir, dass wenn - wie es z. B. der Autor der deutschen Wikipedia-Seite will - ein Einfluss Marcel' auf Mounier bewiesen ist ${ }^{53}$, könnten wir die Wirkung des dialogischen Denkens auch auf den Personalismus und solcher von Personalismus inspirierten Denker wie Paul Ricoeur und Karol Wojtyla ausdehnen.

Schauen wir kurz auf die "Selbstdefinition” dieser Richtung, die in dem „Personalistischen Manifest” Mouniers' gesehen werden kann. Bezüglich der Kritik des Idealismus lesen wir dort: „Es gibt keinen unpersönlichen Geist, kein unpersönliches Geschehen, keinen unpersönlichen Wert, kein unpersönliches Schicksal. Unpersönlich ist nur die Materie" ${ }^{\prime 4}$. Erinnern wir auch daran, dass sich für die Personalisten eine Person ausschließlich durch ihre Beziehung zu einer anderen Person konstituiert und realisiert. Das, was Ebner „das Verhältnis des Ich zum Du” nennt, heißt hier "Gemeinschaft” oder besser „Kommunion” (la communion) der Personen, bzw. einfach „Dialog”. Mounier schreibt:

51 Vgl. G. Marcel, Die geistige Entwicklung Ferdinand Ebners, op. cit., S. 111 f. Wir sollen dennoch feststellen, dass ein solcher Christozentrismus, bereits von Buber suggeriert, nicht ganz auf Ebner zutrifft, und wenn schon, dann eher auf der persönlichen, wie es auch Marcel suggeriert, als auf theoretischen Ebene. Vgl. S. Zucal, Ferdinand Ebner..., op. cit., S. 197.

Ebenda.

Vgl. T. Gadacz, Historia..., op. cit., S. 449.

54 E. Mounier, Das personalistische Manifest, Zürich 1936, S. 81. 
„Wir meinen, dass die Gemeinschaft in das Herz der Person eingepflanzt ist und einen integralen Teil ihrer Existenz bildet" ${ }^{25}$. Diese Grundlage ermöglicht Mounier u. a. eine Unterscheidung zwischen Personalismus und Individualismus. Wir sehen also, dass auch das personalistische Denken dem dialogischen Prinzip entspricht.

\subsection{Katholische Theologie: Karl Rahner, Hans Urs von Balthasar und Vaticanum II}

Unter den katholischen Theologen, die von dem dialogischen Gedanken inspiriert sind, werden auch so bedeutende Persönlichkeiten wie Karl Rahner und Hans Urs von Balthasar genannt. Betrachten wir zuerst den ersten von ihnen. Während weit bekannt ist, dass Rahner in seiner philosophischen Anthropologie von Heidegger profitiert, wissen es nicht viele, dass sein Konzept des „Hörers des Wortes” von Ebner stammt ${ }^{56}$. A. Raffelt meint, dieses kleine Werk - „das letzte philosophische Werk Rahners” - habe, auch wegen seines programmatischen Titels, die Theologie, besonders die in der zweiten Hälfte des 20. Jahrhunderts stark und dauerhaft beeinflusst ${ }^{57}$. Wenn wir dieses Werk nur mit einiger Kenntnis Ebners lesen, können wir es leicht, trotz der Heidegger'schen und Rahner'schen Terminologie und gewisser Regeln der Gedankenentwicklung, als Anwendung des dialogischen Prinzips auf die Beziehung zu Gott interpretieren.

Betrachten wir die Grundverfassung des Menschen, wie Rahner sie präsentiert: zu ihr gehört natürlich das „bei-sich-Sein”, aber nicht nur: „Der Mensch ist die absolute Offenheit für alles Sein [hier: Gott oder

55 Ebenda, S. 96. („Nous trouvons donc la communion insérée au coeur même de la personne, intégrante de son existence même").

56 Vgl. K. Rahner, Hörer des Wortes: Zur Grundlegung einer Religionsphilosophie, München 1941. Nach dem mündlichen Bericht von Prof. Silvano Zucal, damals Dissertant in Innsbruck, auf seine Frage, woher er seine Formulierung vom „Hörer des Wortes”, genommen hat, „wohl auch von Heidegger?” hat Rahner geantwortet: „Nein, ich habe sie von Ebner”. Bericht von Prof. Silvano Zucal, wohnhaft in Trento (Italien) von 17.03.2009.

57 Vgl. A. Raffelt, H. Verweyen, Karl Rahner, München 1997, S. 41. 
Mensch - K. S.], oder, um dieses in einem Wort zu sagen, der Mensch ist Geist”58. Auf diese Weise ist „die Grundverfassung des Menschen eine apriorische absolute Transzendenz auf das Sein überhaupt", deswegen wird der Mensch auch Geist genannt ${ }^{59}$. Auch die Rolle des Wortes in der „Kommunikation” des Seins wird von Rahner angesprochen: „Das Sein ist gelichtet, ist «Logos», und kann so im Wort geoffenbart werden" ${ }^{0}$. Das Wort verbindet sich bei Rahner ebenfalls mit Liebe: „Der Mensch ist in dem Maße horchend auf das Reden oder Schweigen Gottes, als er sich in freier Liebe dieser Botschaft des Redens oder Schweigens des Gottes der Offenbarung öffnet" 61 .

Rahner geht es dabei um den konkreten, realen Menschen, was er durch die Betonung seiner Materialität und Historizität unterstreicht ${ }^{62}$. Es ist aber nicht unsere Absicht, eine vollständige Übereinstimmung zwischen dem Denken Rahners und Ebners zu behaupten. Seine Einstellung zu Ebner charakterisiert vielleicht am besten Rahner selbst in seiner Rezension des von uns bereits zitierten „sehr ebner'schen” Buches „Welt und Person” von Romano Guardini:

Da und dort wird man vielleicht nicht ganz mitgehen: so, wenn [...] Person und christliche Person ähnlich wie bei Ebner oder Brunner zu nahe aneinander gerückt werden. Aber immer liest man dankbar diese Versuche, die uns die Grundkategorien einer christlichen Lehre von Menschen neu und ursprünglich sehen lehren ${ }^{63}$.

In unserem Kontext spielt auch Hans Urs von Balthasar eine sehr wichtige Rolle, ein Denker, der sich nicht nur offen auf die „Dialogiker”,

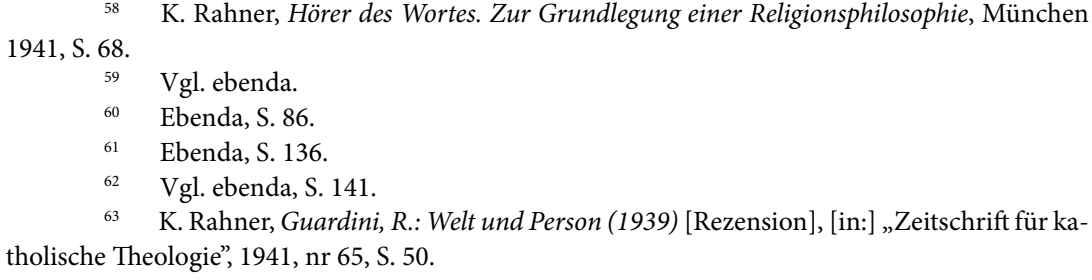
1941, S. 68

K. Rahner, Hörer des Wortes. Zur Grundlegung einer Religionsphilosophie, München 
darunter auch auf Ebner, beruft, sondern auch ausgesprochen dialogisch denkt. Urs von Balthasar ist natürlich ein Theologe, aber in seiner Überzeugung sind auch die Dialogiker „Philosophen, die um der Durchführung ihres Gedankens selbst der Theologie bedürfen. Philosophie und Theologie sind hier "wechselweise auf einander angewiesen»" 64 .

Nach Zucal wird die ganze Dialektik, welche die Balthasarianische Theologie durchwirkt, in dem dialogischen Schlüssel entwickelt ${ }^{65}$. Die Dialogizität ist z. B. in der Trinität sichtbar und findet ihr Bild im Menschen, dem imago trinitatis. Auch bei Balthasar wird der Mensch prinzipiell als eine „angesprochene”, fundamental in Beziehung zum menschlichen und göttlichen Du stehende Person gesehen. Deswegen bringt gerade die Dialogik den Menschen „an die Schwelle der Antwort auf die Frage "Wer bin ich" "66. Dennoch, bei seinem ganzen Respekt und seiner Sympathie zur Dialogik, ist auch Urs von Balthasar weit davon entfernt, sich mit irgendwelchen ihrer Repräsentanten zu identifizieren. Das Denken Ebners hält er z. B. für „schmal und steil”" und er versucht es in seinem opus magnum (Theodramatik/Theologik) zu überwinden.

Wenn dermaßen bedeutsame katholische Denker dem dialogischen Prinzip folgten, kann es nicht mehr verwundern, dass auch in den Dokumenten des Vaticanum II, ein Einfluss des dialogischen Denkens feststellbar ist ${ }^{68}$. Man sieht es wohl am Beispiel der Veränderung des Verständnisses von Offenbarung zwischen Vaticanum I und II. Besonders die Konstitution Die Verbum, wie es Josef Ratzinger betont, stellt ein neues Verständnis der göttlichen Offenbarung dar, das im Laufe des letzten Jahrhunderts „maßgeblich durch den dialogischen Personalismus Ferdinand Ebners, Martin Bubers und anderer geprägt wurde" 69 .

${ }^{64}$ H. U. von Balthasar, Theologik, op. cit., S. 45.

65 Vgl. S. Zucal, Ferdinand Ebner..., op. cit., S. 206 f.

${ }_{66}$ H. U. von Balthasar, Theodramatik, Bd. 1: Prolegomena, Einsiedeln 1973, S. 587 f.

67 Ders., Theologik, op. cit., S. 51.

${ }_{68}$ Vgl. J. Chapel, Das Wort im Sakrament der Beichte, [in:] Logos und Dialogos. Ferdinand Ebner Symposion Brixen, Wien 2012, S. $181 \mathrm{f}$.

69 J. Ratzinger, Dogmatische Konstitution ..., op. cit., S. 506. 
Die Darstellung der göttlichen Offenbarung bricht mit ihrer legalistischen Darstellung als Dekret göttlicher Entscheidungen und stellt ihr das Verständnis des göttlichen Willens als sacramentum entgegen: „eine sakramentale Sicht [...], die Gesetz und Gnade, Wort und Tat, Botschaft und Zeichen, die Person und ihre Äußerungen in der umfassenden Einheit des Mysteriums ineins schaut"70. Dieses Mysterium ist aber nichts anderes als Christus selbst, es ist eine Person. Hier findet also das dialogische Bild des Menschen seinen Ausdruck:

Die göttliche Ansprache: „Nicht mehr Knechte nenne ich euch, sondern Freunde" [geschieht] hier und heute und [...] [will] uns zur Antwort führen. So wird sichtbar, wie der Offenbarungsgedanke zugleich ein Menschenbild entwirft: der Mensch als das dialogische Wesen, das im Hören auf Gottes Wort dem Präsens Gottes gleichzeitig wird und in der Gemeinschaft des Wortes die Wirklichkeit empfängt, die dieses Wort unteilbar ist: die Gemeinschaft mit Gott selbst ${ }^{71}$.

Wir haben gezeigt, dass die Dialogphilosophie, wenn wir sie nicht als eine Doktrin einer bestimmten Gruppe von Denkern verstehen, sondern als das dialogische Denken, oder genauer als Denken nach dem dialogischen Prinzip auffassen, in der katholischen Kirche so wertvoll erschien, zugleich "neu und ursprünglich” (K. Rahner), dass sie mit der Zeit zum Kern der philosophisch-theologischen Überlegungen vorgedrungen ist. Auch wenn sie vielleicht keine ausdrücklich dominierende Rolle spielt, bietet sie dennoch eine interessante Alternative zu den durch gegenwärtige Philosophie ausgearbeiteten Lösungen. Mehr noch, wenn wir die Vision des Urs von Balthasar annehmen, können wir eine besondere Symbiose zwischen dem am dialogischen Prinzip orientierten und dem strikt theologischen Denken feststellen: erst das dialogische Bild des Menschen ermöglicht es uns, uns zur Antwort auf

\footnotetext{
70 Ebenda, S. $506 \mathrm{f}$.

${ }_{71}$ Ebenda, S. 507.
} 
die Frage „Wer bin ich” (auch im Bezug auf Gott) zu nähern, während die "rein philosophische" Dialogik, welche auf einer rein zwischenmenschlichen Begegnung begründet wird, stabiler Grundlagen zu entbehren scheint ${ }^{72}$.

Diese Entwicklung wäre wahrscheinlich nicht möglich gewesen ohne den anfänglichen Impuls, der von Ebners Überlegungen zum Logos des Johannesevangeliums ausgegangen ist. Dieser "katholische Meister” (wie ihn Rosenzweig nennt ${ }^{73}$ ), obwohl kritisch gegenüber jeglichem, auch kirchlichem Monologismus, ruft seit beinahe hundert Jahren zur Beschäftigung mit dem realen Menschen auf und mit seiner Existenz nach dem dialogischen Prinzip, nicht im Sinne der reiner Reflexion, sondern um ihm die Fülle des Lebens zu ermöglichen.

72 Vgl. H. U. von Balthasar, Theodramatik, op. cit., S. 590.

73 Vgl. F. Rosenzweig, Der Mensch und sein Werk. Gesammelte Schriften I: Briefe und Tagebücher, Bd. 2, Haag 1979, S. 889. 